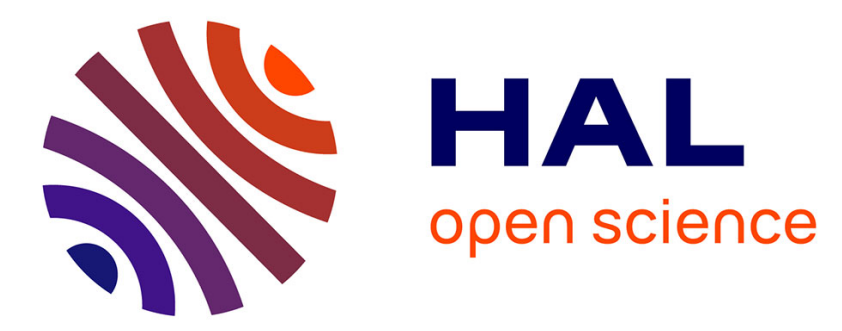

\title{
JPSearch: New international standard providing interoperable framework for image search and sharing
}

Kyoungro Yoon, Youngseop Kim, Je-Ho Park, Jaime Delgado, Akio Yamada, Frederic Dufaux, Ruben Tous

\section{- To cite this version:}

Kyoungro Yoon, Youngseop Kim, Je-Ho Park, Jaime Delgado, Akio Yamada, et al.. JPSearch: New international standard providing interoperable framework for image search and sharing. Signal Processing: Image Communication, 2012, 27, pp.709 - 721. 10.1016/j.image.2012.05.001 . hal-01435345

\section{HAL Id: hal-01435345 \\ https://hal.science/hal-01435345}

Submitted on 13 Jan 2017

HAL is a multi-disciplinary open access archive for the deposit and dissemination of scientific research documents, whether they are published or not. The documents may come from teaching and research institutions in France or abroad, or from public or private research centers.
L'archive ouverte pluridisciplinaire HAL, est destinée au dépôt et à la diffusion de documents scientifiques de niveau recherche, publiés ou non, émanant des établissements d'enseignement et de recherche français ou étrangers, des laboratoires publics ou privés. 


\title{
JPSearch: New international standard providing interoperable framework for image search and sharing
}

\author{
Kyoungro Yoon ${ }^{\mathrm{a}, *}$, Youngseop Kim ${ }^{\mathrm{b}}$, Je-Ho Park ${ }^{\mathrm{c}}$, Jaime Delgado ${ }^{\mathrm{d}}$, Akio Yamada ${ }^{\mathrm{e}}$, Frederic Dufaux ${ }^{\mathrm{f}}$, Ruben Tous ${ }^{\mathrm{d}}$ \\ ${ }^{a}$ School of Computer Science and Engineering, Konkuk University, 1 Hwayang-Dong, Kwangjin-Gu, Seoul, 143-701, Korea \\ ${ }^{b}$ Department of Electronic Engineering, Dankook University, 152, Jukjeon-ro, Suji-gu, Yongin-si, Gyeonggi-do, 448-701, Korea \\ ${ }^{c}$ Department of Computer Science, Dankook University, 152, Jukjeon-ro, Suji-gu, Yongin-si, Gyeonggi-do, 448-701, Korea \\ ${ }^{d} D M A G$, Universitat Politecnica de Catalunya, Jordi Girona, 1-3, 08034, Barcelona, Spain \\ ${ }^{e}$ Information and Media Processing Laboratories, NEC Corp. 1753 Shimonumabe, Nakahara, Kawasaki 211-8666 Japan \\ ${ }^{f}$ Laboratoire de Traitement et Communication de l'Information (LTCI) - CNRS UMR 5141 Télécom ParisTech, F-75634 Paris Cedex 13, France
}

\begin{abstract}
For the last 15 years, there has been various research and great advances in the field of content-based search and retrieval of images. In this paper, we present an overview of the JPSearch Standard, which is a recently published set of international standards providing interoperable framework for image search and sharing. The JPSearch standard is composed of six parts, which are Part 1: System framework and components, Part 2: Registration, identification and management of schema and ontology, Part 3: Query format, Part 4: File format for metadata embedded in image data (JPEG and JPEG 2000), Part 5: Data interchange format between image repositories, Part 6: Reference software. The Part 1 provides motivation and overview of the JPSearch framework. The Part 2 supports interoperability among various metadata specifications and social tagging using the specification of core metadata and translation rule description language. The Part 3 provides powerful image query language for interoperability among multiple image databases. The Part 4 specifies a file format based on JPEG and JPEG 2000 file format in which arbitrary number of metadata description of a image can be embedded. The Part 5 supports interoperability in interchanging images with metadata between image repositories. Finally, the Part 6 provides reference and utility softwares based on which the JPSearch compliant systems can be easily developed. In this paper, summaries and usage examples for each part of the JPSearch standard are presented.
\end{abstract}

Keywords: JPEG, Image Search, Image Sharing, Social Tagging, Metadata

\section{Introduction}

Since the introduction of Query-by-Image-Content [1], there has been various research and great advances in the field of content-based search and retrieval of images [2]. The effort of research on image search and retrieval was greatly increased with the popularity of digital cameras and phone cameras. Some efforts, which may not cover the complete scope of JPSearch Frame-

\footnotetext{
${ }^{*}$ Corresponding author

Email addresses: yoonk@konkuk. ac.kr (Kyoungro Yoon), wangcho@dankook . ac.kr (Youngseop Kim), dk_jhpark@dankook.ac.kr (Je-Ho Park), jaime.delgado@ac.upc.edu (Jaime Delgado), a-yamada@da.jp.nec.com (Akio Yamada), frederic.dufaux@telecom-paristech.fr (Frederic Dufaux), rtous@ac.upc.edu (Ruben Tous)

URL: http://mbm.konkuk.ac.kr (Kyoungro Yoon)
}

work, related to parts of JPSearch standard can be found in [3] [4] [5] [6] [7] [8].

In 2003, 50 million units of digital cameras were sold worldwide according to Photo Marketing Association (PMA) (www.pmai.org) marketing research, and the digital camera market was growing rapidly. Stimulated by the explosion of digital camera market and JPEG images, JPEG working group of ISO/IEC started to explore the JPSearch project with the objective of specifying metadata format and related functionalities to support flexible and efficient still image search, in April 2004.

The idea of developing standard for image search and retrieval became concretely structured over time. Providing an abstract framework of search architecture that decouples the components of image search and a standard interface between these components became

May 1, 2012 
the objectives of the JPSearch standard. The idea of searching images using metadata and developing related functionalities had been exchanged with MPEG working group. It evolved over time resulting in the idea of producing one technical report, four parts of technical specifications and finally a reference software from 2007 to 2011.

The development of the JPSearch standard aimed at solving three main problems as the following:

- Lack of the ability to reuse metadata

When a user annotates a collection of images using one system, it is almost impossible to adopt second type of annotation system with additional functionality without redoing the annotation. Also, when a community of users are annotating a single shared image, which we call a social tagging, it is very hard to merge all the annotations into a single unified metadata.

- Lack of a common query format and search semantics

Search is an essential functionality of large image repositories. However, the various systems providing image search functionality do not provide a common way of specifying a search. Therefore, a query given by a user may be interpreted in different ways. To provide homogeneous interface for the users and make searching over various image repositories consistent, a common query format and search semantics should be defined.

- Lack of a common format for handling context in searching

The terms that people use for query may have different meaning from user to user and from case to case. The specific meaning of a term used may depend on the context of use. For the system to understand the context of a specific user there should be a common format of specifying the context of a certain term being used in the query.

About four years have been spent to provide a concrete specification of standard framework for image search systems. As a result, one technical report of JPSearch Part 1 and technical specifications of five parts are either published or on their way to publication as international standards, which are JPSearch Part 2: Registration, identification and management of schema and ontology [9], JPSearch Part 3: Query format [10], JPSearch Part 4: File format for metadata embedded in image data (JPEG and JPEG 2000) [11], JPSearch Part 5: Data interchange format between image repositories
[12] and JPSearch Part 6: Reference software and conformance [13].

Part 1 is the technical report providing an overview of the JPSearch standard with some usage scenarios.

Part 2 specifies three main tools to support interoperability between image descriptions based on different metadata schemas. The core metadata serves two purposes. One is to provide definitions of essential metadata describing images. The other is to provide a reference in describing third party metadata with help of translation rules. The JPSearch Translation Rules Declaration Language (JPTRDL) is defined to enable specifying guidelines for translating third party defined metadata schema to core metadata. In addition, management tools are defined to register, update and retrieve third party metadata with translation rule to the JPSearch metadata authority, so that users can understand metadata instances which are not defined using JPSearch Core Metadata.

Part 3 specifies message format in XML schema to be sent and received between information requestors (clients) and information providers (database servers). These messages are divided into three types of input query, query output, and query management tools. Input query specifies the syntax and semantics for composition of queries by specifying complex combinations of query conditions, such as boolean combinations of various query conditions, mix of query conditions of various media types as well as relevance feedback. Query output specifies the expected message format and information to be included in the returned data from the responder. Query management tools helps users or applications to select the service by defining message formats describing service capabilities of individual service provider.

Part 4 specifies file formats based on JPEG file format and JPEG 2000 file format to carry metadata with the images. These file formats allow multiple metadata to be embedded inside the JPEG file or JPEG 2000 file. Social tagging is supported by enabling multiple occurrences of metadata inside an image file.

Part 5 specifies a data interchange format for the exchange of image collections with metadata which can be provided at the individual image level as well as the collection level. By defining the interchange format, JPSearch allows synchronization between image repositories, independent of platform or device types.

Part 6 provides reference and utility software to show normative use of the JPSearch tools and various use cases.

Figure 1 shows the architecture of JPSearch Framework. When a query is composed, it can be represented 


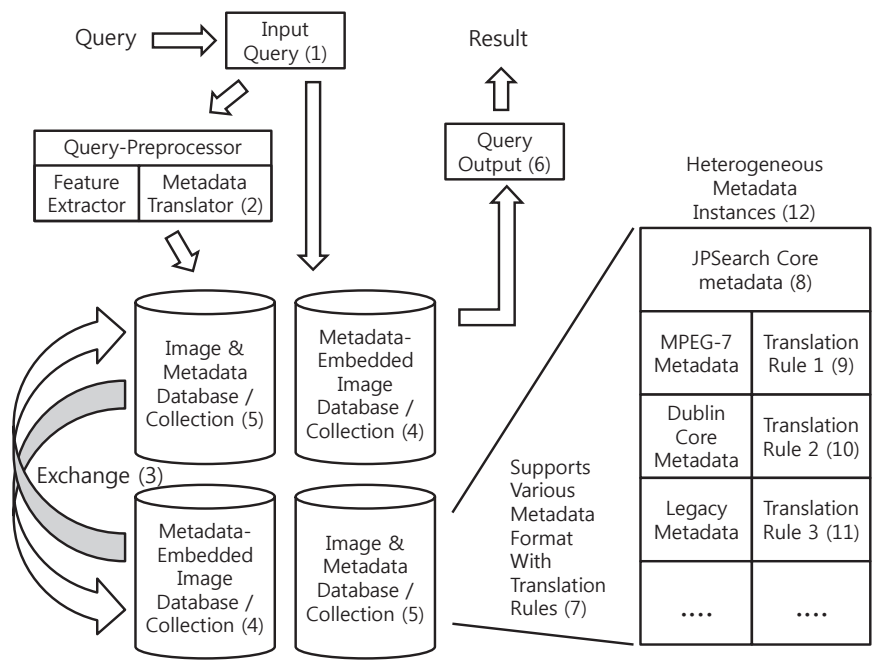

Figure 1: Structure of JPSearch Framework

by the Input Query of JPSearch Query Format defined in Part 3, and is marked as (1) in the figure. The query can be based on the metadata or an image implementing query-by-example. When an example image is given as a query, either a feature extracted from the image or a metadata can be given as the query condition. If the metadata given in the query is defined by a metadata schema other than the JPSearch Core metadata, it can be translated into an instance of the JPSearch core metadata, shown in (8), by following the interpretation of the translation rules defined in Part 2 and represented by (2). The query can be sent directly or after going through the query preprocessor to the database. The database can be a collection of images and metadata as shown in (5) or a collection of images in which metadata is embedded using the file format defined in Part 4 as shown in (4). The database or the collection of images with metadata can be exchanged using the interchange format defined in Part 5 as shown in (3). The image file format defined in Part 4 supports heterogeneous metadata instances as shown in (12) to be embedded in images in JPEG or JPEG2000 format. These heterogeneous metadata can have essential interoperability through the translation rules defined in Part 2 as shown in (7)(9)(10)(11). The processed query result (6) is formed into a query output as defined in Part 3 and returned to the user.

In this paper, details of Part 2, Part 3, Part 4, Part 5 and Part 6 are provided in the following sections. Conclusion and discussions are provided in the last section.

\section{JPSearch Part 2: Registration, identification and management of schema and ontology}

\subsection{Introduction}

JPSearch Part 2 concerns how users can search and retrieve images that include metadata within their physical image files. The images under consideration can be, moreover, spread out multiple repositories that might be managed by a number of independent authorities or systems. Here, the metadata schemas that are used for images do not need to be identical among the different systems as well as the systems maintaining the images do not need to understand the metadata schemas of other systems. In order to provide interoperable search functionality among the different repositories or systems, one common metadata schema, JPSearch Core Metadata, is utilized for delivering a user's query in an understandable manner among the systems that process search related operations using the different metadata schemas.

To retrieve images that satisfy a user's demand, a query referring information in images metadata would be composed by exploiting particular data elements or fields that are only known to the user. The composed query is submitted to a designated interface in order to be spread out the query to the systems that cooperate with the interface carrying the user's original intention. At this point, the submitted query is respectively transformed into a number of different queries that can be understood by the target system(s) regarding metadata syntax, particularly metadata schema terms. In order to 
support this scenario, JPSearch Part 2 provides the following:

- Description of JPSearch Core Metadata Schema in XML,

- Rules for the publication of machine-readable translations between proprietary metadata schema and JPSearch Core Metadata Schema, and

- Rules for the registration and request of metadata schema and its translation rules or links to them.

\subsection{JPSearch Core Metadata Schema}

JPSearch Core Metadata Schema mainly consists of four types: PersonNameType, PublisherType, SourceType and JPSearchCoreType. The PersonNameType is used for the simple description of human beings within the image or image regions. The PublisherType describes publisher related information that can be any public or private organization or person. The SourceType is devised for the description of sources of an image especially when the image is created from an artificial work or product, such as painting, book, video and so on, that exists before the creation of the image.

The JPSearchCoreType is devised in order to describe the information about an image in metadata and contains the most important fields that are necessary for image maintenance work flow such as identifier, modifier, creator, publisher, time related values, description, source, keyword, title, rating and collection related values, GPS position and region related information. In order to support such a role of JPSearchCoreType, additional types are provided in the form of XML types. These additional types are used for description of ownership right, place, person, organization, event, object inside an image, region of interests, region location, external description, rating term definition, image identifier related information and GPS location.

If a system supporting a particular metadata schema wants to be included within an image retrieval operation, the information regarding the system's metadata schema as well as its correspondence relationship concerning JPSearch Core Metadata Schema is required to be registered at a JPSearch Registration Authority (JPSRA).

\subsection{Metadata and Translation Rule Maintenance}

The list of the registration authorities would be provided at a site maintained by ISO/IEC JTC1. The registering system or organization needs to keep the registered schema and translation rules available online to allow validation of external independent applications. When JPSRA verifies the provided information and approves the necessary correctness for JPSearch compliance, JPSRA supports requests for registered information and for replacement of existing information. All these processes are managed by using XML encoded form that is described in SchemaManagment element. For example, registration of a metadata schema or an application needs to provide the following information:

- provider information such as name, description, contact information, and provider capability,

- schema information such as description, name, version, identifier, and location, and

- translation rules that specifies the rules which are necessary for reformulating a query from the reference metadata model to the registered target metadata model.

After review of the registered application, according the review results, an identifier would be assigned to the registered metadata and translation rules or necessary processing would be started in order to complete the registration process.

The schema registration and maintenance process utilize the SchemaManagment element that include information according to the undergoing process: registration, request of registered information or replacement of existing information. Moreover, the corresponding result for a specific operation also utilizes the similar XML structure.

\subsection{Translation Rule Description}

A translation rule describes correspondence relationship between JPSearch Core Metadata Schema and a registered metadata schema such as oneto-one, one-to-many, or many-to-one relationship in the context of XML element level. To support the relationship description, JPSearch defines three types: OneToOneFieldTranslationType, ManyToOneFieldTranslationType, and OneToManyFieldTranslationType. The position of a particular XML element is described by using XPath expression. Moreover, JPSearch metadata translation rule system provides mechanism for complex selection of elements in correspondence relationship definition by using a type FilteredSourceFieldType that supports regular expression, variable usage, position based binding and list based binding. 


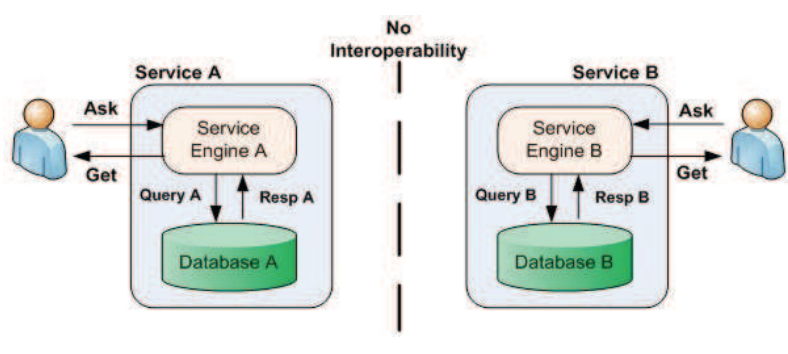

Figure 2: Situation without interoperability

\subsection{Examples of Using Part 2}

Here, we are going to demonstrate two scenarios regarding utilization of JPSearch Core Metadata Schema and Translation rules for interoperability. For the first scenario, we assume that there exist two sites, Service A and Service B, that maintain two distinctive image databases, Database A and Database B, and provide image retrieval functionality from the respective databases. We also assume that each service site maintains image related information by using a specific information structure, schema. Hence one site does not understand the schema used in the other site. As a result, there exists no interoperability between two service sites. Figure 2 demonstrates the above-mentioned situation.

JPSearch Core Metadata Schema here, plays a role for interoperability among the sites. If each site can understand and maintain the mapping rules between Core Metadata Schema and its own information schema based upon semantic correspondence in the constituent element level, the two service sites are able to communicate in terms of image retrieval request and resulting image group. For example, a user who tries to retrieve images from both sites submits a request through a specific site, say Service A. Then, an agent process, say Agent A, that accepts the request written in Service A's specific schema structure generates two queries for the local service engine and the remote agent in Service B.

The Service Engine A processes the submitted query and responses to the agent with a result without further additional operation. Agent A here translates the submitted original query to a query by using Core Metadata Schema elements referring the information that is locally maintained. This translated message described using JPSearch Core Metadata Schema is being sent to Agent B that translates the received query into the query using Service B's schema. After this translation, Agent B submits the translated query to Service Engine B that generates a result. This result will be sent to Agent A through Agent B. After collecting two results from both sites, Agent A merges the results and generates a inte-

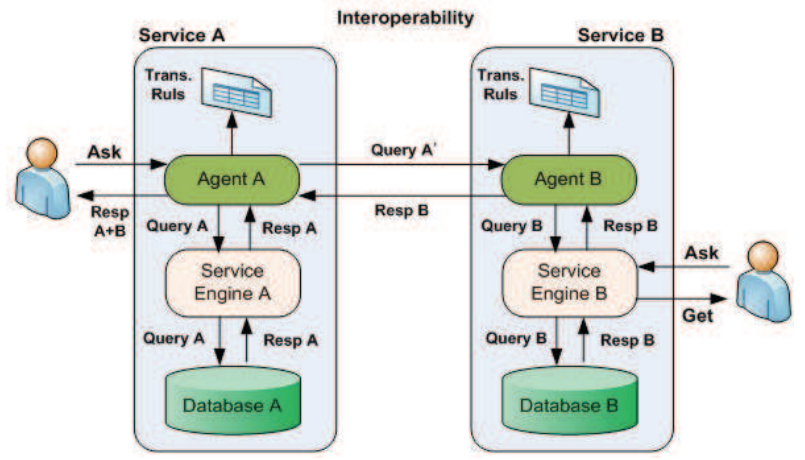

Figure 3: Situation with interoperability

grated response for the user. The reverse case can be processed by switching the workflow's direction. This situation is demonstrated in Figure 3.

The above example assumes two service sites. In the case of more than two sites, the second scenario can be a general case of utilization of JPSearch Core Metadata schema and translations rules. In second scenario, a representative site is known to users as a portal for retrieval of images from multiple image database service sites. The sites that want to be involved in this heterogeneous system need to register their mapping rules between JPSearch Core Metadata and respective schema by using the specified manner that is described in JPSearch Part 2.

When all the members in the system become JPSearch compliant after demanded registration process, the representative site can replicate the user's request by using registered correspondence relationship information that is maintained in the representative site. As in the first example, the correspondence relationship information is described in the context of correspondency between a specific metadata schema and JPSearch Core Metadata Schema in the level of elements taking into account the semantic similarity. The representative site also takes responsibility of merging the results from multiple member sites. This scenario is illustrated in Figure 4.

\section{JPSearch Part 3: JPSearch Query Format}

One of the key elements in JPSearch is the query format, which is specified in Part 3 of ISO/IEC 24800. The tools of the JPEG Query Format (JPQF) are adaptations of ISO/IEC IS 15938-12 (MPEG Query Format, or MPQF) for the still images domain. The MPQF was developed by the MPEG Committee (ISO/IEC JTC1 


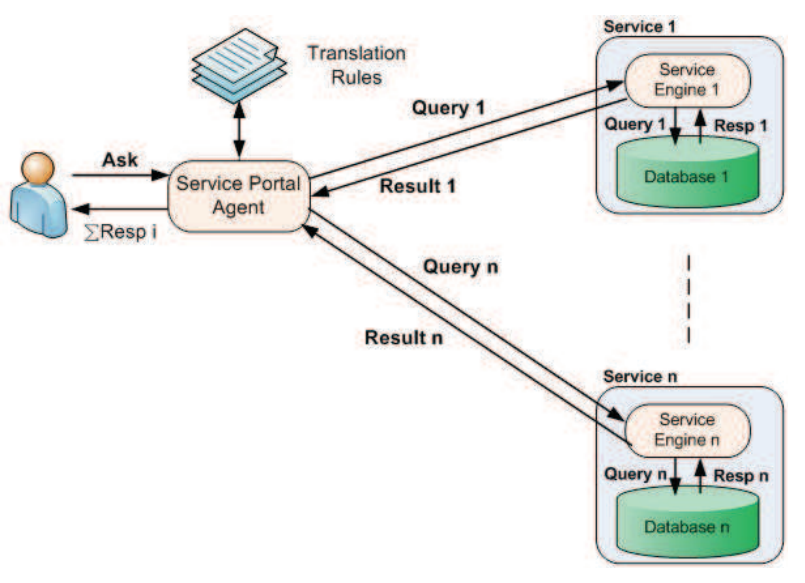

Figure 4: Interoperability among multiple service sites

SC29/WG11) with the objective of providing a standardized interface for multimedia content retrieval systems in order to solve the interoperability problem in distributed multimedia search and retrieval.

MPQF allows combination of Data-Retrieval-like conditions (XPath, XQuery) with InformationRetrieval-like conditions (Query-by-Example, etc.). In other words, combined query of text-based keywords or metadata and image examples is allowed. It is an XML application with one XML schema describing messages both from requester to responder (Input Query Format) and from responder to requester (Output Query Format), and also management messages (service properties, etc.). MPQF and JPQF instances are XML documents that can be validated against MPQF and JPQF schema, respectively. Although it was initially developed in the MPEG-7 context (in fact, it is Part 12 of the MPEG-7 standard), it is metadata-neutral.

In turn, the goal of JPQF is to facilitate and unify access to search functionalities in distributed digital image repositories. To achieve this goal, the JPQF standard also specifies precise input and output parameters to express requests, and uniform client side processing of result sets. Moreover, the management component of JPQF covers searching and the choice of the desired services for retrieval. For this purpose, the standard provides a means to describe service capabilities and to undertake service discovery.

Hence, JPQF is essentially an XML-based query language that defines the format of the queries and replies exchanged between clients and servers in a distributed image search and retrieval system (see Figure 5). There are two main benefits in the standardization of such a language:

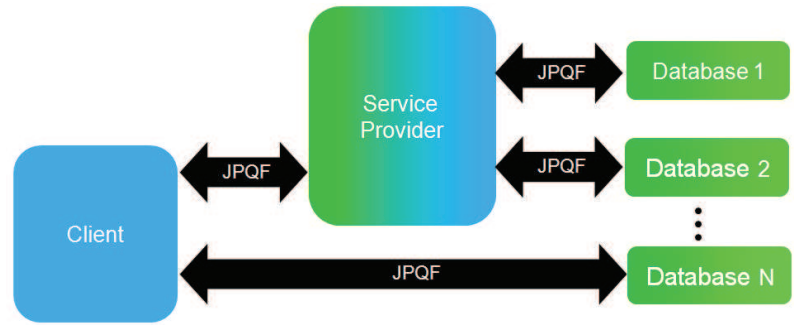

Figure 5: Possible scenario for the use of the JPEG Query Format

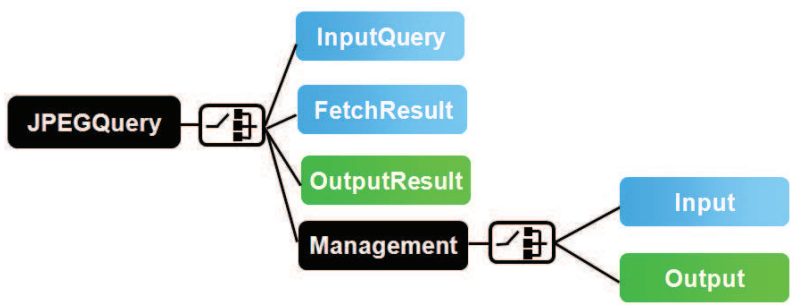

Figure 6: Schema overview of the uppermost elements of the JPQF

- Interoperability between parties in a distributed scenario (e.g. content providers, aggregators and clients).

- Platform independence (which also offers benefits for non-distributed scenarios).

As a result, developers can build applications handling image queries independently of their internal format and even of the offered service. In the example of Figure 5, the Client application only needs to know about JPQF in order to query external databases that may have, or not, JPQF as their native query interface.

JPQF instances are XML documents that can be validated against the JPQF XML Schema. A JPQF instance always starts with the JPEGQuery element as the root element and has the InputQuery element, the FetchResult element, the OutputResult element or the Management element as the top level elements (See Figure 6). JPQF instances with the InputQuery element or the OutputResult element are the usual requests/responses of an image multimedia search process. The InputQuery element is used if the document is a request, and the OutputResult element is used if the document is a response. The InputQuery may have QueryCondition element to specify the search criteria of the database through various QueryType type elements such as QueryByMedia, QueryByDescription, QueryByFreeText and QueryByRelevanceFeedback, to name a few, and their Boolean combinations. The InputQuery also 


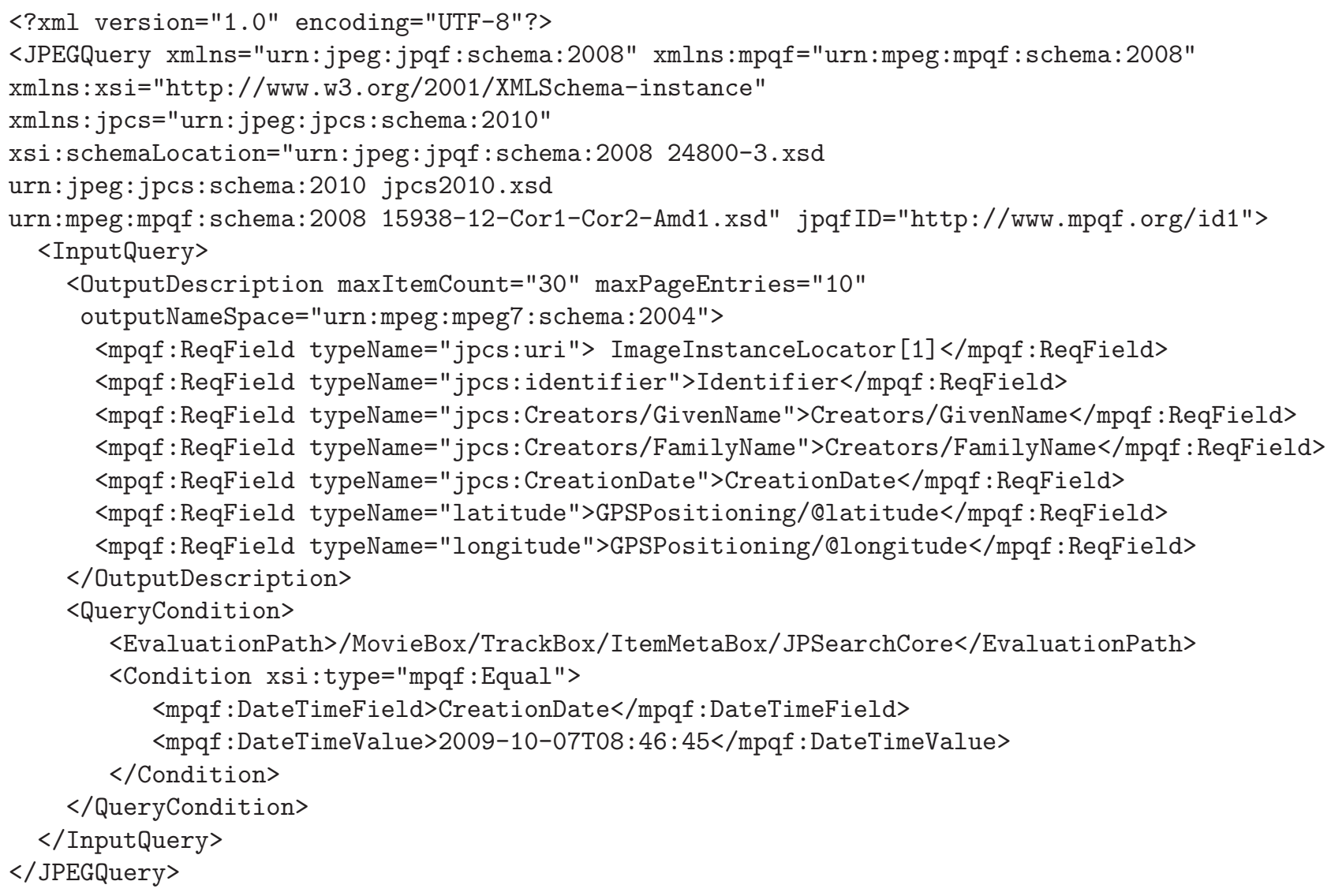

Figure 7: Example of Input Query in JPQF

may have OutputDescription element to specify the format of the message to be returned as the search results. A special query input constitutes the FetchResult element, which is also a top level element, to be used in asynchronous mode for the collection search results. Alternatively, below the root element, a JPQF document can include the Management element. Management messages (which can be both requests and responses) provide a means for requesting service-level functionalities such as discovery of multimedia services or other kinds of service provision, interrogating the capabilities of a service, or configuring service parameters.

Taken from the current Working Draft of the second edition of JPSearch Part 1, the example in Figure 7 shows a JPQF query which asks for images created on 7th October 2009 at 8:46:45. It is worth mentioning the use of the OutputDescription element (requesting some features for the expected answer) before the QueryCondition. In this example, maximum of 30 items are reqested by using the
maxItemCount attribute, in 10 items per page by using the maxPageEntries attribute. Among all the possible elements to be returned, this query requests specifically for URI of the image location, identifier, given name and family name of the creator, creation date, GPS position of the place that the picture is taken by quantifying the ReqField elements of the OutputDescription element. For the query condition, this query specifies that the CreationDate field value of DateTimefield type specified in the JPSearchCore element should be matched to "7th October 2009 at 8:46:45 AM."

The Figure 8 shows an snippet of an example instance of JPQF showing a combined query of free text and example image. In this example, the JPEG images similar to the image given by the url of db.jpeg.org/imagedata001.jpg and matches the text description of Seoul is requested. In this example, two different type of query, i.e. QueryByFreeText and QueryByMedia is combined by a boolean operator of AND. As shown in this example, it is very straightforward to compose a combined query of different query 


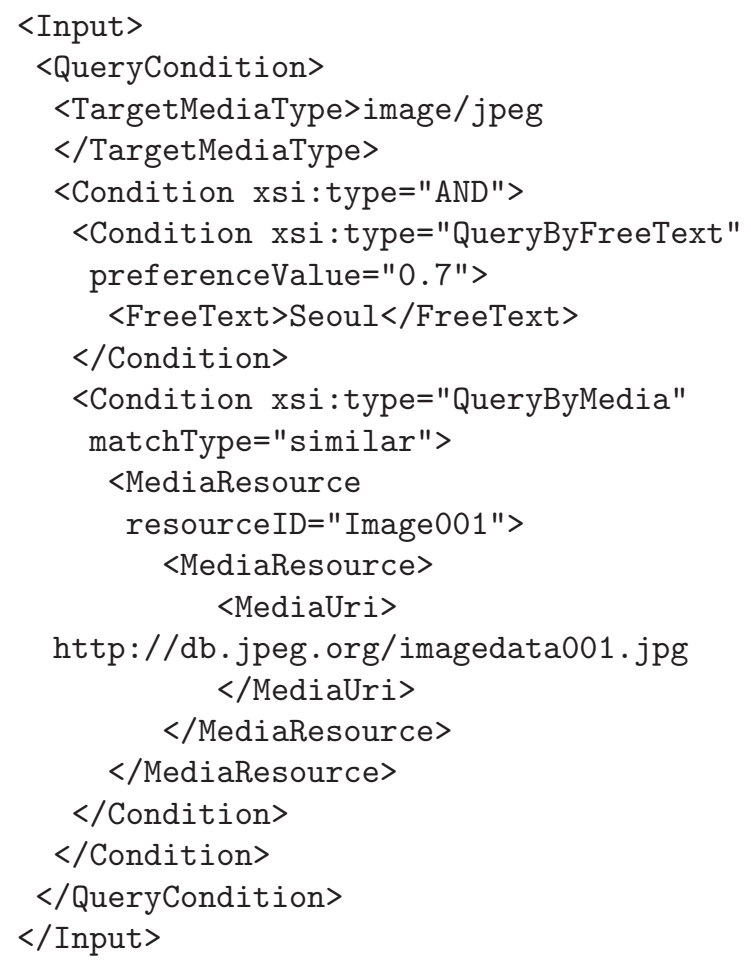

Figure 8: Example of Combined Query in JPQF

types

The Figure 9 shows an snippet of an example instance of JPQF showing a relevance feedback query. In this example, the user selected that result number 4,8 and 10 from the query result with id IDofPreviousQuery are relevant to the intended query. In this example, other part of query is intentionally not shown to give focus on the condition part of the relevance feedback query.

\section{JPSearch Part 4: File Format}

\subsection{Overview}

JPSearch Part 4 focuses on the interoperability of image metadata and enables metadata portability between image repositories as well as various kinds of image handling applications.

One of the biggest problems in existing image management system is the lack of the ability to reuse metadata. A user, who wants to establish well-organized image archive supporting image retrieval, is forced to make a heavy investment to annotate images. There are so many efforts for automatically annotating images, however, in the current market, they are not very popular due to the lack of accuracy and flexibility. After spending a long time for annotation, he/she may find

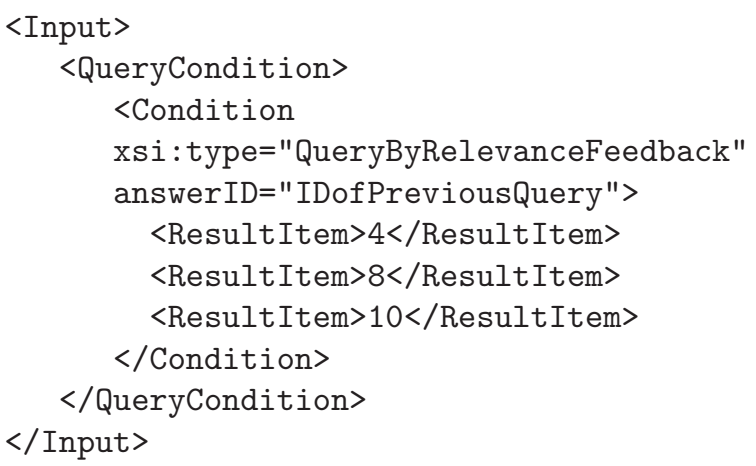

Figure 9: Example of Relevance Feedback Query in JPQF

another problem that he is unexpectedly locked-in a service provider since there are no easy way to carry out his annotation efforts into other services. JPSearch Part 4 provides metadata carriage capability by embedding the metadata in image data itself so that the user never worries about the lost of his achievements when he wants to move his content from a specific service provider to another. The benefits of the usage of Part 4 could be concluded that

- The standardized format allows metadata carriage along with content itself.

- The standardized format is completely compatible with corresponding existing file formats so that any systems/tools/applications used in a current market are fully applicable to JPSearch file format.

- The standardized format can carry any kinds of metadata instances. If their schema is registered into JPSearch registration authority, the instances are understandable by other users.

- The standardized format allows multiple instantiation of metadata to support social tagging for the use of images in a community. Multiple instantiation of metadata of different schema is also available to use an image for various applications.

There are two types of JPSearch file format; one is fully compatible to JPG (JPEG) file format and the other is to J2K (JPEG2000) file format. Figure 10 shows the overall structure of these two types. Both formats have a common format-independent blocks named as JPSearch metadata block (JPS-MB) to carry metadata instances. A JPS-MB is a binary bitstream and designed to carry multiple instances of Elementary Metadata (JPS-EM), which is a basic carriage unit of a metadata instance as described in 4.4 


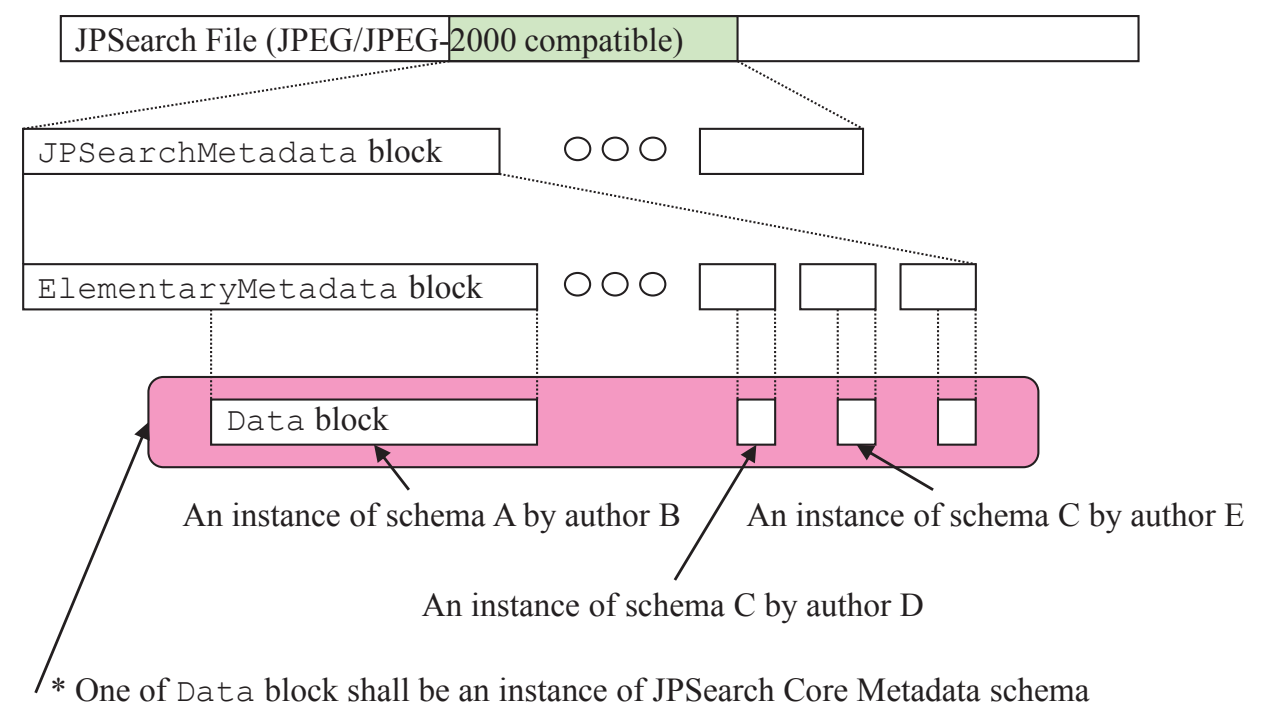

Figure 10: Concept of JPSearch file format

\subsection{JPS-MB carriage in JPEG compatible file format}

JPS-MB is stored in JPEG file format using application marker segments, APP3, as shown in Figure 11. Multiple instantiation of APP3 block is allowed to store a large size metadata instance in a file since an APP3 segment has a limitation of size. A JPS-MB shall not be divided across several APP3 segments. When the size exceeds the limitation, several sets of JPS-MB, each of which has different JPS-EM, shall be employed. An APP3 segment can have only one JPS-MB inside.

\subsection{JPS-MB carriage in JPEG-2000 compatible file format}

JPS-MB is stored in JPEG-200 file format using UUID (Universally Unique Identifier) boxes with specific universally unique identifier as shown in Figure 12. Since JPEG-2000 file format allows multiple JPEG2000 codestreams inside, two different types of UUID boxes can be instantiated. The UUID box at the toplevel in the file structure is used to describe globally applicable metadata and those in codestream-layer headers are used to describe the corresponding local codestream. If local information is different from the global one, the local description overwrites the global one.

Figure 11: Structure of JPEG compatible JPSearch file format

\begin{tabular}{|c|}
\hline JP2 Signature box \\
\hline JP2 File Type box \\
\hline JP2 Header box \\
\hline Codestream header box \\
\hline UUID box (JPSearch (local)) \\
\hline Compositing layer header box \\
\hline UUID box (JPSearch (local)) \\
\hline UUID box (JPSearch (global)) \\
\hline contiguous codestream box \#i \\
\hline
\end{tabular}

Figure 12: Structure of JPEG-2000 compatible JPSearch file format

For example, if there are several codestreams basically capturing identical scene with different coding parameters or coding schemes in a single JPEG-2000 file, the set of metadata describing the scene captured in the provided JPEG-2000 file may be provided in the UUID box at the top-level and individual set of metadata specific to a single codestream with a certain coding parameters or coding scheme may be provided in the UUID box of corresponding codestream header box, respectively. 


\subsection{Metadata carriage using JPS-EM}

A JPS-EM is the most fundamental structure to describe a metadata instance according to a specific schema by a single metadata author. It consists of

(1) identifier of the used schema,

(2) various descriptive information of metadata creation process such as creation/update date/time, author information, and confidence of the description, and

(3) metadata itself.

Any type of metadata schema can be used even if the schema is not registered into the JPSearch Registration Authority, so a user can embed his/her metadata using his/her original schema without losing interoperability of a file. Note that if the schema is not registered, the JPS-EM, unfortunately, may not be understandable by other users, although this unknown (unregistered) metadata block can still be securely delivered along with image itself (in compressed image bitstream).

If another instance using different schema is required, the user can easily add another JPS-EM into a JPS-MB. For an example, a metadata for medical purpose can coexist with a metadata for content sharing purpose in a single file entity. Also, if another user has different view from the existing metadata instance, he can instantiate additional JPS-EM to describe his own idea as a metadata. Therefore, in the case of social tagging joining 10 participants, there can be a compressed image data and ten JPS-EMs in a single file.

\section{JPSearch Data Interchange Format Between Im- age Repositories}

\subsection{Scope}

Part 5 of JPSearch [12] provides a data interchange format for the exchange of image collections and respective metadata between JPSearch compliant repositories. The metadata can be at the level of the image or an image collection. By providing a solution for the carriage of image collections and associated metadata between compliant devices and platforms, the JPSearch data interchange format enables the synchronization of repositories in order to facilitate simple and fully interoperable exchanges across different devices and platforms.

The JPSearch data interchange format should enable the easy and reliable transfer of data between different hardware and software systems. In particular, it should support functions such as
- exchange of data between JPSearch repositories on different devices and platforms,

- consolidation of metadata generated on different systems,

- transfer of data to a newer and better system,

- consolidation of selected data to a centralized repository, and

- archive of data in a format which will survive current products.

The JPSearch data interchange file format encompasses both internal or external resources, namely encoded image bitstreams, along with collection-level and item-level metadata, namely entity of descriptive data of image data.

\subsection{File format and resource}

The JPSearch data interchange file format encapsulates all the collection-level and item-level metadata into a valid XML descriptor in a single text-based format. The aim is to enable easy exchange of metadata between repositories.

The collection-level and item-level metadata should follow the interchange format schema as defined in the specifications (see Sec. 5.3.2). Besides a plain-text XML representation, the descriptive metadata can optionally be stored using BiM [14].

Only JPEG-conforming [15], JPEG 2000conforming [16] and JPSearch-conforming codestreams may be used as internal or external resources in the JPSearch data interchange file format. The ImageDataType type allows linking between metadata and each corresponding internal or external resource when used within the binary file format, or just external resources when used within the XML metadata interchange format.

Internal resources shall be stored in InlineMedia of ImageDataType, an instance of an image codestream encoded in hex binary of base64 binary format. Alternatively, external resources can be identified by MediaUri of ImageDataType, which describes where the image file, to which these metadata refer, is located.

As a special case, a file can have all images defined as external resources. As a result, the file only contains metadata (collection-level and item-level).

\subsection{Schema and metadata}

In this section, we describe the metadata which is supported by the JPSearch data interchange format. 


\subsubsection{JPSearch collection metadata schema}

We first discuss the schema that facilitates the composition of XML metadata descriptions about image collections, for the purpose of information exchange between image repositories. Specifically, the type hierarchy of the schema and the root element are described.

The Collections element serves as the root element of the collection metadata schema. The root element shall be used as the topmost element when collections metadata appears in an independent way. This applies when collections metadata appears within the top-level metabox of the binary file format.

The CollectionsType type serves as the root element of the collection metadata part of the metadata interchange format schema.

The CollectionType type allows expressing metadata related to one image collection. It includes descriptive elements from the JPSearch Core Schema in JPSearch Part 2.

\subsubsection{JPSearch XML metadata interchange format schema}

We now discuss the schema that facilitates the interchange of XML metadata descriptions about images and collections. The following description tools are specified: The type hierarchy of the schema and the root element.

The ImageRepository element serves as the root element of the XML metadata interchange format schema. It corresponds to the movie box as defined in the ISO base media file format [17].

The ImageRepositoryType type allows expressing metadata related to images and image collections. It is composed by zero-to-many CollectionsMetadata elements, and one-to-many Image elements. It corresponds to the movie box type as defined in the ISO base media file format [17].

The CollectionsMetadataType type allows expressing metadata related to image collections. It is composed by multiple Collection elements from the CollectionType defined in the JPSearch collection metadata schema.

The ImageType type allows expressing metadata related to one image.

The ImageDataType type allows linking between metadata and each corresponding internal or external resource when used within the binary file format, or just external resources when used within the XML metadata interchange format.

The ImageMetadataType type allows expressing metadata related to one image (item level). It includes

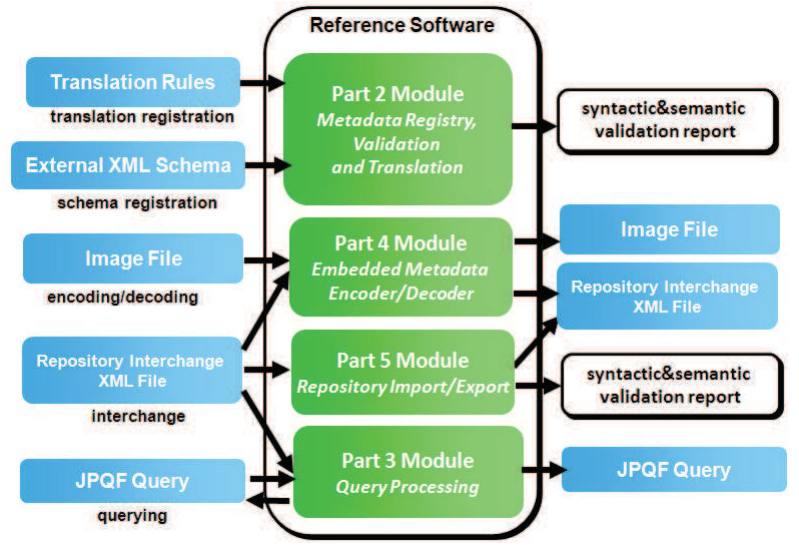

Figure 13: JPSerach reference software architecture

descriptive elements from the JPSearch Core Schema in JPSearch Part 2, but also offers the possibility to include metadata formalized according to external or userdefined schemas (e.g. MPEG-7).

\section{JPSearch Reference Software and Applications}

\subsection{JPSearch Reference Software}

JPSearch provides reference software in order to help implementers interpreting the ISO/IEC 24800 specifications, and also to enable them determining whether their products or systems are conformant to the standard. The JPSearch reference software, which is specified in Part 6, provides a specific implementation that behaves in a conformant manner. However, other implementations that conform to ISO/IEC 24800 are possible that do not necessarily use the same algorithms or the same programming techniques. The JPSearch reference software operates on and generates conformant JPSearch metadata and image files. It is entirely written in the Java programming language and it is divided in four different modules, one for each normative part of the standard (Parts 2, 3, 4 and 5). These software modules are defined in a way that they can be used independently or combined to form a basic JPSearch compliant system. Figure 13 depicts the architecture of ISO/IEC 24800 reference software.

The Part 2 module (Metadata Registry, Validation and Translation) allows registering external metadata schemas, validating metadata instances and defining cross-schema metadata translations. This module performs syntactic and semantic schema/translation validation, allowing implementers determining whether their schemas and translations are conformant to the 


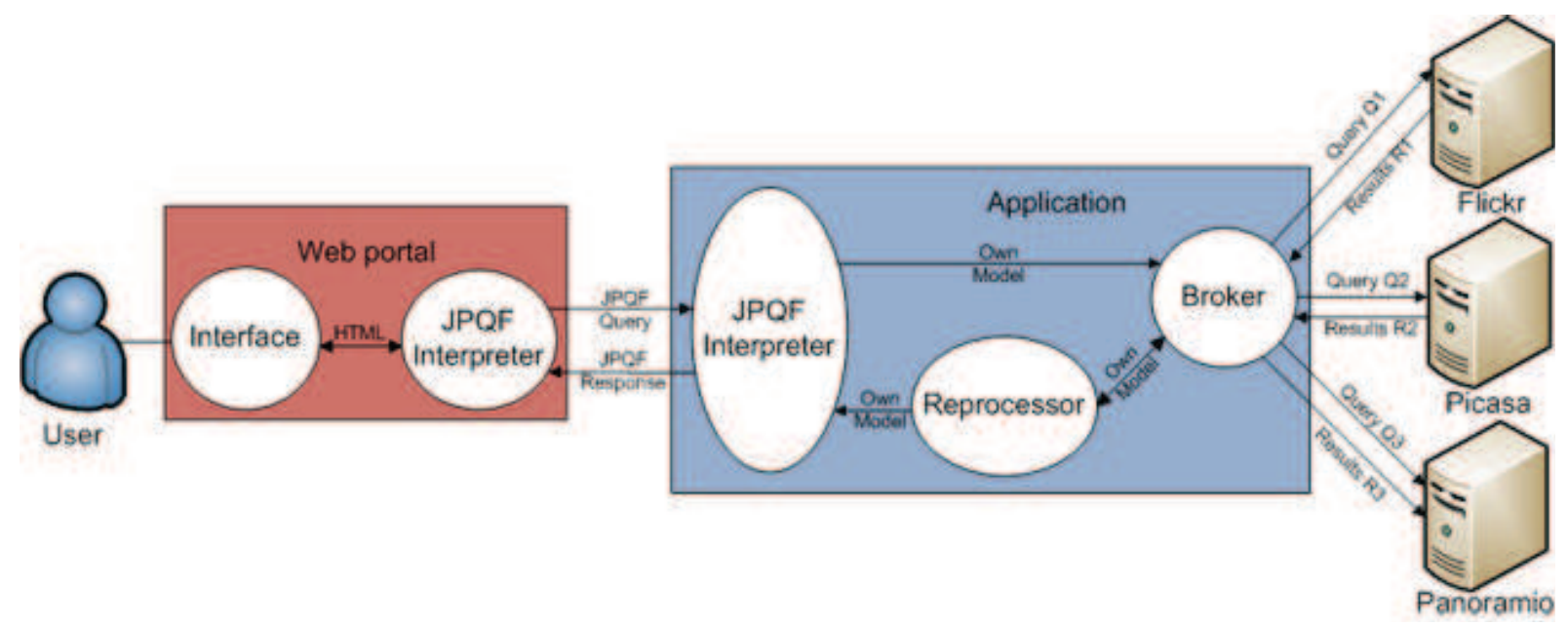

Figure 14: Federated image search system architecture

rules defined in ISO/IEC 24800-2. The Part 3 module (Query Processing) processes JPSearch queries as defined in ISO/IEC 24800-3 (informally referred as JPSearch Query Format or JPQF) against a given image repository (provided by the user in the form of an ISO/IEC 24800-5 file). This module performs query validation and accepts queries containing boolean operators, XPath expressions, arithmetic and comparison expressions, sorting and grouping. The results of query evaluation are also returned as a JPQF message. The Part 4 module (Embedded Metadata Encoder/Decoder) allows extracting/annotating metadata embedded within an image file. This module accepts either an already annotated image file or a pair (image file, ISO/IEC 24800-5 file) and returns either an ISO/IEC 248005 file or an annotated image file respectively. Part 5 module (Repository Import/Export) allows importing/exporting metadata from an internal repository. It accepts ISO/IEC 24800-5 files and performs syntactic validation.

\subsection{JPSearch Applications}

JPSearch is a recent standard, but there are already some real applications which are taking profit from it. One example is the federated image search system developed by the DMAG (Distributed Multimedia Applications Group), a research group of the Computer Architecture Dept. of the Universitat Politcnica de Catalunya (UPC BarcelonaTech) [18]. The aim of this project, further described in [19], is to provide the ability to search images, from a central point, on different servers such as Panoramio, Picasa or Flickr, simultaneously. The system is compliant with Parts 2 and 3 of the JPSearch standard. It receives ISO/IEC 24800-3 (JPQF) queries addressing metadata in ISO/IEC 248002 format (extended with some EXIF fields, like the camera make and model) and rewrites them once for every metadata format that is supported (Panoramio, Picasa and Flickr in the first public version). In order to ensure that the results are correct, they are reprocessed (or filtered) based on the search constraints, whenever it is possible. This filtering also expands the search capabilities of each server because it allows searches that would not be possible without doing it. Figure 14 shows the system architecture. A demo of the system can be found in [20].

Another example usage of ISO/IEC 24800 is the BIOPSEARCH system [21], a content based medical image retrieval application specialized in optical biopsies. An optical biopsy is an optic diagnostic method capable to analyze the tissue in surface and in deepness without the need to extract it from the body. The system assists physicians and other medical personnel in the interpretation of optical biopsies obtained through confocal laser endomicroscopy (CLE). CLE is a novel technique for intravital microscopy during ongoing gastrointestinal endoscopy. However, most gastroenterologists are not trained to interpret mucosal pathology, and histopathologists are usually not available in the endoscopy suite. BIOPSEARCH may greatly facilitate CLE data base management and diagnosis. The system, also developed by DMAG, allows users navigating and searching over an image database containing optical biopsies of the human colon. Users are able to retrieve information about precedent diagnostics by providing an example CLE image for content based image 


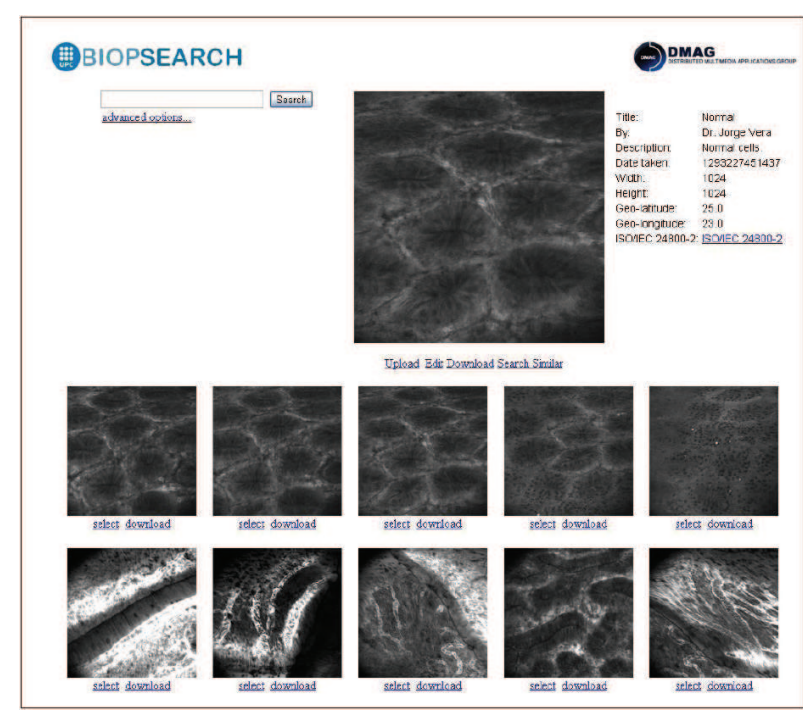

Figure 15: Screenshot of the BIOPSEARCH demo GUI client

retrieval (CBIR), by using keywords, or by filtering different fields for structured retrieval. The system is conformant with ISO/IEC 24800-2 and ISO/IEC 24800-3. Figure 15 shows a screenshot of the system's web frontend.

\section{Conclusion and Discussions}

In this paper, we introduced a new international standard of JPSearch, which provides a unified framework for interoperable search and sharing of images. Some of the major advantages of JPSearch are as follows:

- JPSearch provides tools for interoperable interpretation of heterogeneous metadata supporting social tagging. By registering their own schema and translation rules to the authority, based on the JPSearch core metadata and translation rule description language, people can add their own metadata such as key words or text description to the existing images and share the images with metadata;

- JPSearch provides unified messages between client and servers for query and result, called Query Format, to allow independent development of applications and standardized interfaces enabling simple aggregation service of multiple database services;

- JPSearch provides interoperable file format, within which arbitrary number of metadata can be embedded, based on JPEG and JPEG2000 file format, and provides straightforward binding mechanism of image and corresponding metadata. Using the JPSearch file format, multiple instances of metadata can be embedded inside the image file and transferred between devices without losing information;

- JPSearch defines interchange format for exchange of data between JPSearch image repositories.

For semantic interoperability of metadata within the JPSearch framework, the JPSearch Ad Hoc Group of ISO/IEC JTC1 SC29 WG1 (a.k.a. JPEG) is currently considering extension of JPSearch standard by including ontology related technology.

\section{Acknowledgement}

This article is a collaborative effort of editors of JPSearch standard. The authors would like to thank members of the JPEG committee who contributed to the development of JPSearch standard.

\section{References}

[1] M. Flickner, H. Sawhney, W. Niblack, J. Ashley, Q. Huang, B. Dom, M. Gorkani, J. Hafner, D. Lee, D. Petkovic, D. Steele, P. Yanker, Query by image and video content: the qbic system, Computer 28 (9) (1995) 23 -32. doi:10.1109/2.410146.

[2] S. K. Shandilya, N. Singhai, A survey on: Content based image retrieval systems, International Journal of Computer Applications 4 (2) (2010) 22-26.

[3] J. Tesic, Metadata practices for consumer photos, Multimedia 12 (3), IEEE (2005) 86-92.

[4] B. N. Lee, W.-Y. Chen, E. Y. Chang, A scalable service for photo annotation, sharing, and search, Proceedings of the 14th annual ACM international conference on Multimedia (2006)

[5] Exif Version 2.2 Digital Still Camera Image File Format Standard (Exif) Version 2.2, Japan Electronics and Information Technology Industries Association (2002).

[6] N. Adami, A. Boschetti, R. Leonardi, P. Migliorati, Embedded indexing in scalable video coding, Multimedia Tools and Applications 48 (1), (2010) 105-121

[7] C. Morand, J. Benois-Pineau, J.-P. Domenger, J. Zepeda, E. Kijak, C. Guillemot, Scalable object-based video retrieval in HD video databases, Sig. Proc.: Image Comm. 25 (6), (2010) 450465

[8] M. Mrak, J. Calic, A.M. Kondoz, Fast analysis of scalable video for adaptive browsing interfaces, Computer Vision and Image Understanding 113 (3), (2009) 425-434

[9] ISO/IEC 24800-2:2011: Information technology - JPsearch Part 2: Registration, identification and management of schema and ontology, June 2011.

[10] ISO/IEC 24800-3:2010: Information technology - JPsearch Part 3: Query format, April 2010.

[11] ISO/IEC 24800-4:2010: Information technology - JPsearch Part 4: File format for metadata embedded in image data (jpeg and jpeg 2000), Nov. 2010.

[12] ISO/IEC 24800-5:2011: Information technology - JPsearch Part 5: Data interchange format between image repositories, June 2011. 
[13] ISO/IEC 24800-6:2010: Information technology - JPsearch Part 6: Reference Software, Sep. 2010

[14] ISO/IEC 23001-1: Information technology - MPEG systems technologies - Part 1: Binary MPEG format for XML.

[15] ITU-T rec. t.81 - ISO/IEC 10918-1, Information technology Digital compression and coding of continuous-tone still images: Requirements and guidelines.

[16] ITU-T rec. t.800 - ISO/IEC 15444-1, Information technology - JPEG 2000 image coding systems: Core coding system.

[17] ISO/IEC 15444-12, Information technology - JPEG 2000 image coding systems: ISO base media file format.

[18] Distributed multimedia applications group (dmag) website. http://dmag.ac.upc.edu/ (last visited 13rd of April 2011).

[19] P. Toran, J. Delgado, Image search based on a broker approach, 11th International Workshop of the Multimedia Metadata Community.

[20] Distributed image search application demo. http://dmag.ac.upc.edu/standardization/jpeg/demo-broker (last visited 13rd of April 2011).

[21] O. Ferrer, F. Ettorre, X. Santos, T. Zinkl, R. Tous, J. Delgado, Solo-medicine in optical biopsies - a way to practice telemedicine, HEALTHINF 2011 Proceedings of the International Conference on Health Informatics (2011) 26-29. 